\title{
EDITORIAL
}

\section{SOBRE EL ESTUDIO DEL IMPACTO DE INTRODUCIR UNA VACUNA EN EL CALENDARIO DE VACUNACIÓN}

\author{
Carmen Amela Heras
}

Centro Nacional de Epidemiología. Instituto de Salud Carlos III. Madrid. España

El impacto de una vacuna, en términos de reducir la incidencia de la enfermedad y la mortalidad de la población por la misma depende, fundamentalmente, de que exista una vacuna eficaz y segura y de que el país disponga de un programa de vacunación que garantice la consecución y el mantenimiento de coberturas de vacunación elevada en los grupos de población sobre los que se sustenta la transmisión de la infección.

La vacuna conjugada anti Haemophilus tipo b (Hib) fue incorporada al calendario de vacunación infantil en el año 1998, por decisión unánime del pleno del Consejo Interterritorial del Sistema Nacional de Salud celebrado el 15 de diciembre de 1997.

El control de la enfermedad invasiva causada por el Haemophilus influenzae es un objetivo de la Región Europea de la Organización Mundial de la Salud (OMS) recogido en el programa Salud 21: el marco político de salud para todos de la Región Europea siendo la meta que los países mantengan una tasa de incidencia inferior al 1 por 100.000 habitantes.

El programa de vacunación en España, además de las vacunas tradicionales frente a poliomielitis, difteria, tétanos, tos ferina, sarampión, rubéola y parotiditis, ha incorporado en los últimos años la vacuna frente a la hepatitis B, la vacuna conjugada frente a Hib y por último en el año 2000 la vacuna conjugada frente al meningococo $\mathrm{C}$. Estas vacunas constituyen el calendario de vacu- nación infantil y se administran en todas las Comunidades Autónomas.

Para incluir una vacuna en el calendario de vacunaciones es necesario conocer la importancia de la enfermedad (incidencia anual, gravedad y letalidad por grupos de edad), la disminución o modificación de la transmisión de la infección en la comunidad como consecuencia de la introducción de la vacuna, la reducción de costes por la hospitalización y tratamiento, la efectividad de la vacuna en la prevención o en la modificación de la enfermedad, la duración de la protección y la seguridad e inmunogenicidad de la administración simultánea o combinada con otras vacunas. El conocimiento de estos factores permitirá debatir sobre la introducción de la nueva vacuna y las estrategias de vacunación más eficientes para el país.

La eficacia de la vacuna conjugada anti-Hib, en estudios prospectivos, ha mostrado ser al menos del $90 \%$ cuando se administran tres dosis en la primera infancia ${ }^{1,2}$. Esta vacuna actúa reduciendo la tasa de portadores y, por tanto, la transmisión, lo que podría explicar por qué la reducción en la enfermedad invasiva es superior al porcentaje de población vacunada.

Cuando se comercializó la vacuna conjugada anti-Hib, la enfermedad invasiva por $H$. influenzae no estaba incluida en la lista de Enfermedades de Declaración Obligatoria, y por tanto no se conocía la incidencia de la enfermedad. 
A partir del año 1994 algunas Comunidades Autónomas (CCAA) comenzaron a realizar una búsqueda de casos retrospectivamente para estimar la incidencia de la enfermedad. En el año 1996 se realizó un estudio retrospectivo, en 12 CCAA; los casos diagnosticados de enfermedad invasiva por $H$. Influenzae en niños de 0 a 4 años eran buscados en los hospitales públicos, con el objetivo de estimar la incidencia acumulada anual y las complicaciones de la enfermedad en los años 1993-1994, es decir el período prevacunal. La incidencia de enfermedad invasiva encontrada fue de 12,4 por 100.000 habitantes, de estos cuadros clínicos el $60 \%$ fueron diagnosticados de meningitis. La letalidad fue del $4,7 \%$ y se presentaron complicaciones y/o secuelas en el $24,3 \%$ de los casos $^{3}$.

El impacto de la vacunación en los países en los que se ha incluido en el calendario de vacunación ha sido espectacular, con descensos superiores al 90\%. A nivel mundial se ha encontrado un impacto mucho menor, estimándose que solamente el $5,9 \%$ de los casos de meningitis y el $8,5 \%$ de los casos de las manifestaciones clásicas por Hib se previenen en niños de 0 a 4 años de edad ${ }^{4}$. La OMS estima que el $H$. influenzae causa al menos 3 millones de casos de enfermedad severa y entre 400.000 a 700.000 muertes de niños en todo el mundo ${ }^{5}$.

Una vez introducida una vacuna en el calendario vacunal es necesario medir el impacto de la vacunación, como ha realizado la Comunidad Valenciana, en la que estudian la evolución de la incidencia anual de la infección por Hib, encontrando una disminución del 91,5\%, entre el año 1996 y el año 2000 (I.A.: 3,56 al 0,30 por 100.000 habitantes $)^{6}$.

En el período postvacunal la dinámica de la relación entre la incidencia de la enfermedad, las coberturas de vacunación y las reacciones adversas pueden modificarse según se avance hacia la fase de eliminación de la enfermedad y por tanto deben vigilarse. $\mathrm{La}$ vigilancia epidemiológica y sobre todo la investigación de brotes en los períodos postvacunales permite identificar los cambios en la epidemiología de las enfermedades vacunables (cambios en la edad de presentación, modificaciones en el cuadro clínico clásico, mecanismos de transmisión, etcétera) y en ocasiones sugiere cambios en el esquema de vacunación. En enfermedades como la enfermedad invasiva por Hib la vigilancia en hospitales puede ser más útil, ya que la mayoría de los casos ingresan en hospitales y se confirman por laboratorio.

Cuando se introducen en el calendario vacunas de reciente comercialización es importante reforzar la vigilancia de reacciones adversas, ya que hay características que no se detectan en los estudios previos a la comercialización, a consecuencia de los límites en el tamaño de la población estudiada, la duración y la heterogeneidad de la población en la que se realizan los estudios, de forma que efectos adversos raros aparecen posteriormente (intosuspección y vacuna frente al rotavirus).

En resumen, la introducción de vacunas en el calendario de vacunación infantil garantiza, hasta el momento, la consecución de coberturas de vacunación elevadas $y$, en función de las características de la vacuna, alcanzar el control o eliminación de la enfermedad. La introducción de nuevas vacunas debería realizarse en base a un buen conocimiento de las características de la enfermedad en nuestro país y tras su introducción los sistemas de vigilancia de la enfermedad, las coberturas de vacunación y las reacciones adversas deberían reforzarse.

\section{BIBLIOGRAFÍA}

1. Eskola J, Kayhty H, Takala AK, Peltola H, Ronnberg PR, Kela E, Pekkanen E, McVerry PH, Makela $\mathrm{PH}$. A randomized, prospective field trial of a conjugate vaccine in the protection of infants and young children against invasive Haemophilus influenzae type b disease. N Engl J Med 1990; 323: 1381-1387. 
2. Booy R, Hodgson S, Carpenter L, Myon-White RT, Slack MP, Macfarlane JA, Haworth EA, Kiddle M, Shribman S, Clair Roberts J and Moxon ER. Efficacy of Haemophilus influenzae tipe b conjugate vaccine PRP.T. Lancet 1994;344: 362-366.

3. Centro Nacional de Epidemiología. Estudio sobre la incidencia de enfermedad invasiva por Haemophilus influenzae en España. Madrid: Instituto de Salud Carlos III; 1997.

4. Peltola H. Worldwide Haemophilus influenzae tipe $\mathrm{b}$ disease at the beginning of the $21^{\text {st }}$ century: global analysis of the disease burden 25 years after the use of the polysaccharide vaccine and a decade af- ter the advent of conjugates. Clin Microbial Rev 2000: 302-317

5. World Health Organization. Global program for vaccines and inmunization. The WHO position paper on Haemophilus influenzae tipe b conjugate vaccines. Wkly Epidemiol Rec 1998; 73: 64-68.

6. M Goicoechea Sáez, AM Fullana Montoro, P Momparler Carrasco, MJ Redondo Gallego, J Brines Solanes y FJ Bueno Cañigral. Enfermedad invasora por Haemophilus influenzae antes y después de la campaña de vacunación en la población infantil de la Comunidad Valenciana (1996-2000). Rev Esp Salud Pública 2002; 76: 197-206. 